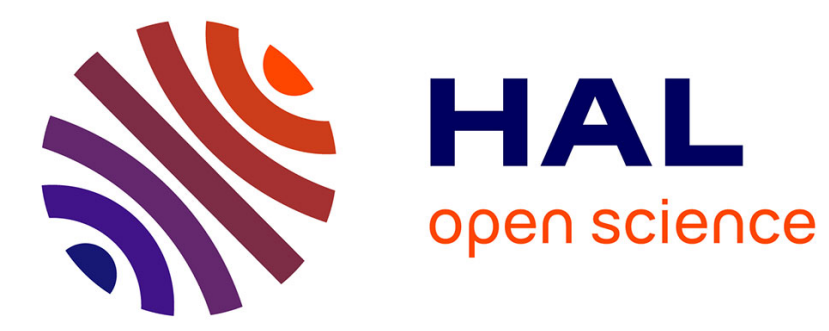

\title{
Interprétation quantique de la modulation de fréquence
}

Nicole Polonsky, Claude Cohen-Tannoudji

\section{To cite this version:}

Nicole Polonsky, Claude Cohen-Tannoudji. Interprétation quantique de la modulation de fréquence. Journal de Physique, 1965, 26 (7), pp.409-414. 10.1051/jphys:01965002607040900 . jpa-00205985

\section{HAL Id: jpa-00205985 https://hal.science/jpa-00205985}

Submitted on 1 Jan 1965

HAL is a multi-disciplinary open access archive for the deposit and dissemination of scientific research documents, whether they are published or not. The documents may come from teaching and research institutions in France or abroad, or from public or private research centers.
L'archive ouverte pluridisciplinaire HAL, est destinée au dépôt et à la diffusion de documents scientifiques de niveau recherche, publiés ou non, émanant des établissements d'enseignement et de recherche français ou étrangers, des laboratoires publics ou privés. 


\title{
INTERPRÉTATION QUANTIQUE DE LA MODULATION DE FRÉQUENCE
}

\author{
-Par Nicole POLONSKY et Glaude COHEN-TANNOUdJi, \\ Laboratoire de Physique de l'École Normale Supérieure, Paris.
}

\begin{abstract}
Résumé. - On étudie le mouvement d'un dipôle magnétique dont on module la fréquence de Larmor en le plaçant dans un champ magnétique $H_{0}+H_{1} \cos \omega t$, modulé en amplitude. Le champ de radiofréquence $H_{1} \cos \omega t$ est traité quantiquement. On montre que l'apparition de fréquences latérales dans le mouvement du dipôle est due à des absorptions et réémissions virtuelles de photons de radiofréquence par le système atomique. Ceci permet d'interpréter certaines caractéristiques de nouvelles résonances magnétiques observées récemment dans des expériences de pompage optique transversal.
\end{abstract}

Abstract. - A study is made of the motion of a magnetic dipole whose Larmor frequency is modulated when placed in an amplitude modulated magnetic field $H_{0}+H_{1} \cos \omega t$. The radiofrequency field, $H_{1} \cos \omega t$, is quantized. It is shown that the sideband frequencies which appear in the motion of the dipole are due to virtual absorption and re-emission of radiofrequency photons by the atomic system. It is then possible to interpret some features of new magnetic resonances observed in transverse optical pumping experiments.

Introduction. - Le but de cet article est de donner une interprétation quantique du phénomène de modulation de fréquence. De façon plus précise, le problème particulier auquel nous nous intéressons est celui d'un dipôle magnétique dont on module la fréquence de Larmor en le plaçant dans un champ magnétique modulé en amplitude $H_{0}+H_{1} \cos \omega t$. La solution classique de ce problème est bien connue $[1, \S 7-5-45]$ : en l'absence du champ de radiofréquence $H_{1} \cos \omega t$, le dipôle précesse à la fréquence de Larmor $\omega_{0} / 2 \pi$ $\left(\omega_{0}=\gamma H_{0}, \gamma\right.$ rapport gyromagnétique) avec une amplitude qu'on prendra égale à l'unité (fig. 1a); le champ de radiofréquence $H_{1} \cos \omega t$ fait apparaître des mouvements supplémentaires (fig. 1b) aux fréquence: latérales $\left(\omega_{0}+p \omega\right) / 2 \pi$ ( $p$ entier positif, nul ou négatif) avec des amplitudes $J_{p}\left(\omega_{1} / \omega\right)\left(J_{p}\right.$ fonction de Bessel d'ordre $p$ avec la convention

$$
\left.J_{-p}=(-1)^{p} J_{\nu} ; \omega_{1}=\gamma H_{1}\right) .
$$

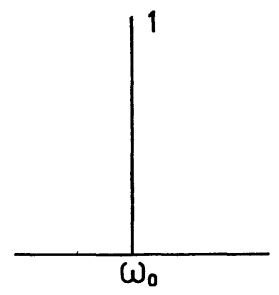

a) $H_{1}=0$

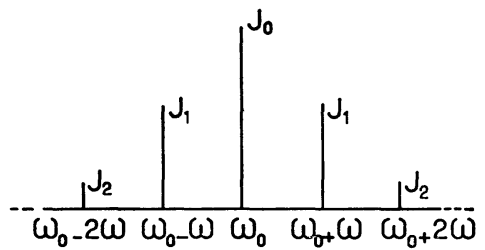

b) $I_{1} \neq 0$

FIG. 1.

Nous avons essayé de donner de ce phénomène une interprétation en termes de photons de radiofréquence en quantifiant le champ $H_{1} \cos \omega t$; ceci fait l'objet de la première partie de ce travail.
L'apparition des fréquences latérales dans le mouvement du dipôle est à l'origine de nouvelles résonances magnétiques observées récemment par divers auteurs $[2,3,4]$, dont une caractéristique essentielle est que leur largeur est indépendante de l'amplitude $H_{1}$ du champ de radiofréquence. Nous présentons dans la deuxième partie de ce travail diverses interprétations physiques de ces nou velles résonances.

\section{Traitement quantique du mouvement $d u$ dipôle magnétique.}

A. Hamiltonien du Système. - Le hamiltonien total du système s'écrit: $\mathfrak{H}=\mathfrak{H}_{0}+\mathfrak{H}_{\mathrm{R}}+\mathfrak{H}_{\mathrm{T}}$. $\mathcal{H}_{0}=\omega_{0} I_{z}$ décrit le couplage Zeeman du dipôle magnétique avec $H_{0}$ supposé parallèle à Oz. Le dipôle est représenté par un spin $I=1 / 2$, dont la composante suivant $\mathrm{Oz}$ est $I_{\mathrm{z}}$. Les deux états propres de $I_{\mathrm{z}}$ sont $\mid \pm>$ séparés par $\omega_{0}$ (nous prenons $\hbar=1$ ). $\mathfrak{H}_{\mathrm{R}}=\omega a^{+} a$ est le hamiltonien $\mathrm{du}$ champ de radiofréquence, $a^{+}$et $a$ étant les opérateurs de création et d'annihilation d'un photon $\omega$. Ses états propres sont désignés par $l n>$, d'énergie $n \omega$.

$\mathcal{H}_{\mathrm{I}}$ est le hamiltonien d'interaction entre l'atome et le champ de radiofréquence. On peut le mettre sous la forme $\mathfrak{H}_{\mathrm{I}}=\lambda I_{\mathrm{z}}\left(a+a^{+}\right)$, $\lambda$ étant un coefficient de couplage réel (la longueur d'onde associée au champ de radiofréquence, étant très grandc devant les dimensions de l'atome porteur du dipôle, on néglige toute dépendance spatiale dans $\mathfrak{H}_{\mathrm{I}}$ ).

Le but du calcul est de déterminer le mouvement de la composante transversale du dipôle, $\left\langle I_{+}(t)\right\rangle$ $\left(I_{+}=I_{\mathbf{x}}+i I_{\mathrm{y}}\right)$. Pour résoudre ce problème, nous commencerons par rechercher les états propres de $\mathcal{H}$. 
B. États propres de $\mathscr{H}_{0}+\mathscr{H}_{\mathrm{R}}$ - Commençons par négliger $\mathcal{H}_{\mathrm{I}}$ : le système atomique et les photons de radiofréquence coexistent sans interagir. Les états propres de $\mathscr{H}_{0}+\mathcal{H}_{\mathrm{R}}$ sont de la forme $|n>| \pm>$, d'énergie $n \omega \pm \omega_{0} / 2$. La figure 2 représente ces niveaux d'énergie groupés en deux colonnes correspondants aux deux états $\mid+>$ et $\mid->$ du dipôle.

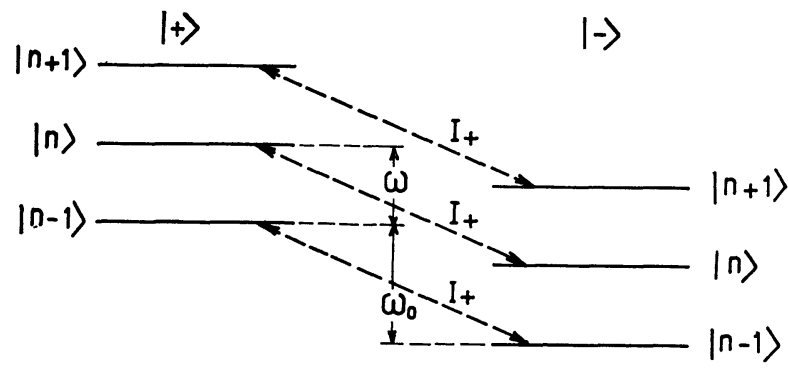

FIG. 2.

Si $E_{\mathrm{i}}, E_{\mathrm{j}} \ldots$ sont les niveaux d'énergie d'un système, toute grandeur physique $G$ de ce système évolue aux fréquences $\left(E_{\mathrm{i}}-E_{\mathrm{j}}\right) / h$ pourvu qu'il existe un élément de matrice non nul de $G$ entre $E_{\text {i }}$ et $E_{\mathrm{j}}$. Dans le cas qui nous intéresse ici, les éléments de matrice de $I_{+}$, représentés en pointillé sur la figure 2, n'existent qu'entre des états $|n\rangle \mid+>$ et $|n>|->$ correspondant au même nombre de photons de radiofréquence $\left(I_{+}\right.$n'agit pas sur les variables de rayonnement). La différence d'énergie entre ces deux états est $\omega_{0}:\left\langle I_{+}\right\rangle$évolue donc à la fréquence $\omega_{0} / 2 \pi$.

C. Effet de He. Traitement de perturbation. - $\mathfrak{H}_{\mathrm{I}}$, étant proportionnel à $I_{z}$, n'a d'élément de matrice qu'entre niveaux d'une même colonne (les photons de radiofréquence ont une polarisation $\pi$ ). Dans chaque colonne $\mathcal{H}_{\mathrm{I}}$ couple donc au premier ordre chaque niveau à ses deux plus proches voisins. Le niveau $|n>| \varepsilon>(\varepsilon= \pm)$ devient ainsi au premier ordre en $\lambda$

$$
\begin{array}{r}
\left|\hat{n}_{\varepsilon}>\right| \varepsilon>=|n>| \varepsilon>- \\
\frac{\varepsilon \lambda}{2 \omega} \sqrt{n+1}|n+1>| \varepsilon> \\
+\frac{\varepsilon \lambda}{2 \omega} \sqrt{n}|n-1>| \varepsilon>.
\end{array}
$$

Nulle au premier ordre, la correction en énergie vaut au deuxième ordre $-\lambda^{2} / 4 \omega$. Elle ne dépend ni de $n$, ni de $\varepsilon$. Tous les niveaux de la figure 2 sont donc déplacés de la même quantités (résultat que nous verrons plus loin être rigoureux à tous les ordres).

Les nouveaux états $\left|\tilde{n}_{\varepsilon}>\right| \varepsilon>$ ne correspondant plus à un nombre bien défini de photons, il apparaît maintenant de nouveaux éléments de matrice pour $I_{+}$, reliant par exemple $\left|\tilde{n}_{\varepsilon}>\right|->$ à $\widetilde{(n+1)_{\varepsilon}}>\mid+>$ et $\left|(n-1)_{\varepsilon}>\right|+>$. Il s'en suit que le mouvement de $<I_{+}>$s'effectue maintenant non seulement à la fréquence $\omega_{0} / 2 \pi$ mais aussi aux fréquences $\left(\omega_{0} \pm \omega\right) / 2 \pi$.

L'apparition de ces nouvelles fréquences d'évolution est donc due au fait que $\mathcal{H}_{I}$ ramène dans l'état $|n>| \varepsilon>$ un peu des états $|n \pm 1>| \varepsilon>$ (expression 1). Ceci peut s'interpréter en disant que l'atome, dans l'état $|\varepsilon\rangle$, absorbe et réémet (ou émet et réabsorbe) virtuellement des photons de radiofréquence, ce qui a pour effet de mélanger un état $|n\rangle$ aux états $\mid n \pm 1>$. Le niveau $\left|\tilde{n_{\varepsilon}}>\right| \varepsilon>$ résultant est déplacé mais non élargi.

En poussant la théorie des perturbations à l'ordre $p$, un état $|n>| \varepsilon>$ devient, par suite d'absorptions et réémissions virtuelles de $k$ photons de radiofréquence, un mélange des états

$$
|n \pm k>| \varepsilon>
$$

( $k$ variant de 0 à $p$ ), ce qui fait apparaître dans le mouvement de $<\cdot I_{+}>$les fréquences

$$
\left(\omega_{0} \pm k \omega\right) / 2 \pi \text {. }
$$

Sous l'effet de ces transitions virtuelles, le mouvement de précession du dipôle autour de $H_{0}$ n'est donc plus uniforme.

D. Calgul rigoureux des états propres de He. - Les états propres de He sont de la forme $\left|\bar{n}_{\varepsilon}>\right| \varepsilon>$ et peuvent être toujours rangés en deux colonnes correspondant à $\varepsilon=+$ ou $-\varepsilon$ étant fixé, c'est-à-dire pour tous les états d'une même colonne, on a :

$$
\mathcal{H}=(\varepsilon / 2) \omega_{0}+V_{\varepsilon}
$$

avec

$$
V_{\varepsilon}=\omega a^{+} a+(\varepsilon \lambda / 2)\left(a+a^{+}\right) .
$$

Les états $\mid \bar{n}_{\varepsilon}>$ sont donc les états propres de $V_{\varepsilon}$. $V_{\varepsilon}$ peut aussi s'écrire sous la forme

$$
V_{\varepsilon}=\omega\left(a^{+}+\frac{\varepsilon \lambda}{2 \omega}\right)\left(a+\frac{\varepsilon \lambda}{2 \omega}\right)-\frac{\lambda^{2}}{4 \omega} .
$$

Introduisons l'opérateur de déplacement :

$$
D(\rho)=\mathrm{e}^{\rho(a+-a)} \quad(\rho \text { réel }) .
$$

On a $[5]$ :

$$
\left\{\begin{array}{l}
D(\rho) a D^{+}(\rho)=a-\rho \\
D(\rho) a^{+} D(\rho)=a^{+}-\rho
\end{array}\right.
$$

$V_{\varepsilon}$ se déduit donc de l'opérateur $\omega a^{+} a-\left(\lambda^{2} / 4 \omega\right)$ par la transformation unitaire $D(-\varepsilon \lambda / 2 \omega)$ :

$$
V_{\varepsilon}=D\left(-\frac{\varepsilon \lambda}{2 \omega}\right)\left[\omega a+a-\frac{\lambda^{2}}{4 \omega}\right] D^{+}\left(-\frac{\varepsilon \lambda}{2 \omega}\right) .
$$

Les états propres $\mid \bar{n}_{\varepsilon}>$ de $V_{\varepsilon}$ se déduisent des états $\mid n>$ par la même transformation :

$$
\left|\bar{n}_{\varepsilon}>=D(-\varepsilon \lambda / 2 \omega)\right| n>=\mathrm{e}^{-\varepsilon \lambda / 2 \omega(a+-a) \mid n>.}
$$


Ils correspondent à la valeur propre

$$
n \omega-\left(\lambda^{2} / 4 \omega\right)
$$

Tous les niveaux de la figure 2 sont donc déplacés de la même quantité : $-\lambda^{2} / 4 \omega$.

E. CALCUL DE $<I_{+}(t)>$ :

a) Expressions rigoureuses. - Nous partirons de la matrice densité du système global, $\rho(t)$, dont l'équation d'évolution $i \mathrm{~d} \rho / \mathrm{d} t=[\mathcal{H e}, \rho]$ s'intègre formellement pour donner

$$
\rho(t)=e^{-i \Im e t} \rho(0) e^{i \Im t t},
$$

$\rho(0)$ étant la matrice densité à l'instant initial. On a alors :

$$
\left\langle I_{+}(t)>=\mathrm{T}_{\mathrm{AR}} \cdot I_{+} \cdot \rho(t)\right.
$$

$\mathrm{T}_{\mathrm{AR}}$ représente l'opération trace par rapport aux variables atomiques et aux variables de rayonnement.

$I_{+}$n'agissant que sur les variables atomiques et n'ayant d'éléments de matrice qu'entre les états $\mid->$ et $\mid+>$, il vient

$$
\begin{aligned}
<I_{+}(t)> & =\sum_{n}<+\left|I_{+}\right|-><-\left.|<n|\right|_{\rho}(t)|n>|+> \\
& =<-\left|\rho_{\mathbf{A}}(t)\right|+>
\end{aligned}
$$

avec

$$
\rho_{A}(t)=\sum_{n}\langle n|\rho(t)| n\rangle=\mathrm{T}_{\mathrm{R} \rho} \rho(t)
$$

$\mathrm{T}_{\mathrm{B}}$ : trace par rapport aux variables de rayonnement.

Les états $\mid \bar{n}_{+}>$(de même que les états $\left.\mid \bar{p}_{-}>\right)$ constituant un système orthonormé complet pour les variables de rayonnement, on a encore :

$$
\begin{aligned}
& <-\left|p_{A}(t)\right|+>
\end{aligned}
$$

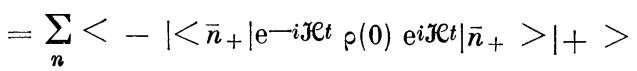

$$
\begin{aligned}
& =\sum_{n, p}<\bar{n}_{+} \mid \bar{p}_{-}>
\end{aligned}
$$

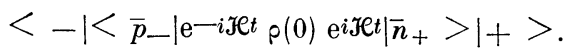

Les états $\left|\bar{n}_{+}>\right|+>$et $\left|\bar{p}_{-}>\right|->$étant respectivement états propres de $\mathcal{H}$ correspondant aux valeurs propres $\omega_{0} / 2+n \omega-\lambda^{2} / 4 \omega$ et $-\omega_{0} / 2+p \omega-\lambda^{2} / 4 \omega$, il vient :

$$
\begin{aligned}
\left\langle I_{+}(t)>=\sum_{n, p}\right. & <\bar{n}_{+} \mid \bar{p}_{-}>\mathrm{e} i\left[\omega_{0}+(n-p) \omega\right] t \\
& <-\left|<\bar{p}_{-}\right| \rho(0)\left|\bar{n}_{+}>\right|+>
\end{aligned}
$$

ou encore, en posant $n-p=q$ :

$$
\begin{gathered}
<I_{+}(t)>=\sum_{n, q}<\bar{n}_{+} \mid(\overline{n-q})_{-}>c^{i i\left(\omega_{0}+q \omega\right) t} \\
<-\left|<(\overline{n-q})_{-}\right| \rho(0) \mid \bar{n}_{+}>1+>
\end{gathered}
$$

Telle est l'expression rigoureuse de $\left\langle I_{+}(t)\right\rangle$. Nous retrouvons bien dans le mouvement de
$\left.<I_{+}(t)\right\rangle$ les fréquences $\omega_{0}+q \omega$, les amplitudes correspondantes faisant intervenir les produits scalaires $<\overline{n_{+}} \mid(\overline{n-q})_{-}>$entre un état de la colonne de gauche et un état de la colonne de droite distants en énergie de $\omega_{0}+q \omega$ (en l'absence de $\mathcal{H}_{\mathrm{I}}$, ces états sont orthogonaux pour $q \neq 0$ ). Nous verrons plus loin que ces produits scalaires ne sont autres, pour $n \gg 1$, que les fonctions de Bessel $J_{q}\left(\omega_{1} / \omega\right)$.

b) Choix de l'état initial $\rho(0)$. - A l'instant initial, $\rho(0)$ est le produit d'une matrice densité atomique $\rho_{A}(0)$ et d'une matrice densité de rayonnement $\rho_{R}(0)$. L'élément de matrice de $\rho(0)$ figurant dans (12) s'écrit alors :

$$
\left\langle I_{+}(0)><\left.(\overline{n-q})_{-}\right|_{\rho_{R}}(0) \mid \bar{n}_{+}\right\rangle
$$

Nous prenons pour état initial du champ de rayonnement l'état quantique qui se rapproche le plus du champ classique $H_{1} \cos \omega t$, c'est-à-dire l'état cohérent de Glauber [5] correspondant :

$$
\left|\psi_{\mathrm{R}}(0)>=\mathrm{c}^{\alpha(a+-a)}\right| 0>=D(\alpha)|0\rangle
$$

$\psi_{\mathrm{R}}(0)>$, état propre de $a$ correspondant à la valeur propre $\alpha$, se déduit du vide par le déplacement $D(\alpha)$. (Nous nous limitons ici à des valeurs réelles de $\alpha$.

Comme l'état $\mid \psi_{\mathrm{R}}(0)>$ correspond au champ classique $H_{1}$, on doit avoir :

$$
<\psi_{\mathrm{R}}(0)\left|\lambda\left(a+a^{+}\right)\right| \psi_{\mathrm{R}}(0)>=\gamma H_{1}=\omega_{1}
$$

ce qui donne

$$
\alpha=\omega_{1} / 2 \lambda
$$

$\mid \psi_{\mathrm{R}}(0)>$ s'écrit aussi :

$\left|\psi_{\mathrm{R}}(0)>=\sum_{n} C_{n}(\alpha)\right| n>$ avec $C_{n}(\alpha)=\frac{\mathrm{e}^{-\alpha^{2} / 2} \alpha^{n}}{\sqrt{n !}}$

$\left[C_{n}(\alpha)\right]^{2}$ est donné en fonction de $n$ par une loi de Poisson (fig. 3) ce qui entraîne les propriétés bien connues:

$$
\begin{gathered}
\left\langle n>=N=\left\langle\psi_{\mathrm{R}}(0)|a+a| \psi_{\mathrm{R}}(0)>=\alpha^{2}\right.\right. \\
\sqrt{\left\langle(\Delta n)^{2}\right\rangle}=v \overline{\left\langle\left(n-\langle n>)^{2}\right\rangle\right.}=\alpha=\vee \bar{N}
\end{gathered}
$$

$\alpha^{2}$ représente donc le nombre moyen $N$ de photons et est, par suite, un nombre extrêmement grand ; ceci entraîne $\alpha=\omega_{1} / 2 \lambda \gg 1 ; \omega$ et $\omega_{1}$ étant du même ordre de grandeur, nous avons aussi $\omega / \lambda \gg 1$.

La dispersion sur $n, \sqrt{\left\langle(\Delta n)^{2}\right\rangle}=\sqrt{N}$, est très grande en valeur absolue, tout en étant très petite en valeur relative :

$$
\checkmark<\overline{(\Delta n)^{2}>} \mid<n>=1 /: \bar{N} \ll 1 .
$$

La matrice densité du rayonnement à l'instant initial s'écrit donc :

$$
\begin{aligned}
\rho_{R}(0) & =\left|\psi_{R}(0)><\psi_{R}(0)\right| \\
& =\mathrm{e}^{\alpha(a+-a)}|0><0| \mathrm{e}^{-\alpha(a+-a) .}
\end{aligned}
$$




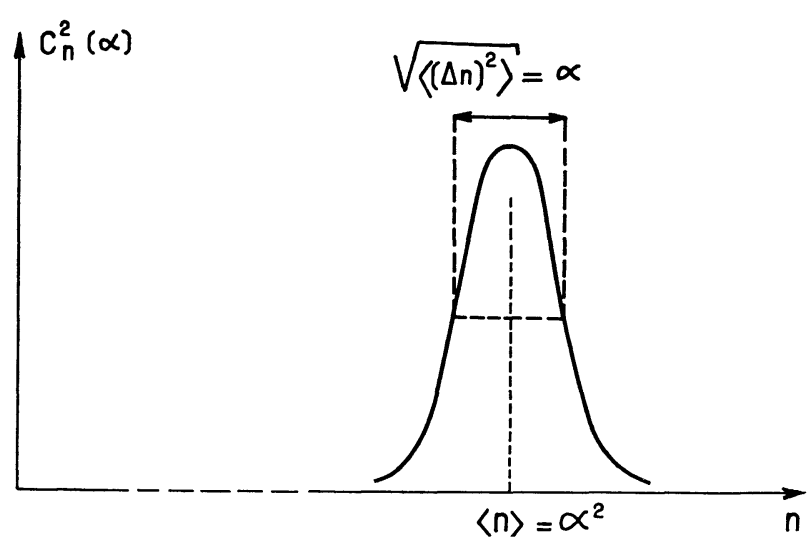

FIG. 3.

Utilisant la relation (7), nous en déduisons :

$$
\begin{aligned}
& <(\overline{n-q})_{-}\left|\rho_{R}(0)\right| \bar{n}_{+}> \\
& =\left\langle n-q\left|\mathrm{e}^{(-\lambda / 2 \omega+\alpha)(a+-a)}\right| 0\right\rangle \\
& <0\left|\mathrm{e}^{(-\lambda / 2 \omega-\alpha)(a+-a)}\right| n> \\
& =C_{n-q}(\alpha-\lambda / 2 \omega) C_{n}(\alpha+\lambda / 2 \omega) \simeq C_{n-q}(\alpha) C_{n}(\alpha) \text {. } \\
& \left\langle I_{+}(t)\right\rangle=\left\langle I_{+}(0)\right\rangle \\
& \sum_{n, q}\left\langle\bar{n}_{+}\right|(\overline{n-q})_{-}>\mathrm{e}^{\mathrm{i}\left(\omega_{0}+q \omega\right) t} C_{n}(\alpha) C_{n-q}(\alpha) .
\end{aligned}
$$

Nous démontrons en appendice la relation suivante, valable pour $n \gg 1$ :

$$
<\bar{n}_{+} \mid(\overline{n-q})_{-}>=J_{q}\left(\omega_{1} / \omega\right)
$$

$J_{q}$, fonction de Bessel d'ordre $q$, devient négligeable pour $q>\omega_{1} / \omega$. D'après la figure $3, C_{n}$ et $C_{n-q}$ ne diffèrent de façon appréciable que pour $q$ de l'ordre de $\sqrt{N}$. Comme $q<\omega_{1} / \omega \ll \sqrt{N}$, on peut dans l'expression (19) remplacer $C_{n-q}(\alpha)$ par $C_{n}(\alpha)$ et obtenir ainsi :

$$
\begin{aligned}
\left\langle I_{+}(t)\right\rangle & =\left\langle I_{+}(0)>\sum_{Q} J_{Q}\left(\omega_{1} / \omega\right) \mathrm{e}^{\mathrm{i}\left(\omega_{0}+q \omega\right) t} \sum_{n} C_{n}^{2}(\alpha)\right. \\
& =\left\langle I_{+}(0)>\sum_{q} J_{q}\left(\omega_{1} / \omega\right) \mathrm{e}^{\mathrm{i}\left(\omega_{0}+q \omega\right) t .}\right.
\end{aligned}
$$

Nous retrouvons ainsi quantiquement le résultat classique énoncé dans l'introduction.

Le calcul que nous venons de présenter ici ne fait que rendre quantitative l'interprétation physique, développée dans le paragraphe I-C, de l'apparition des nouvelles fréquences $\omega_{0}+q \omega$ dans le mouvement de $\left\langle I_{+}(t)\right\rangle$ : le développement limité des fonctions de Bessel à l'ordre $k$ redonne le résultat de la théorie des perturbations au même ordre $k$.
II. Application ; Interprétation d'un nouveau type de résonances magnétiques observées dans un champ magnétique modulé en amplitude. A. DESCRIPTION ET INTERPRÉtATION QVALITATIVE DES NOUVElles RÉSONANGes. - Le pompage optique transversal d'une vapeur atomique placée dans un champ magnétique modulé en amplitude $H_{0}+H_{1} \cos \omega t$ a permis récemment à divers auteurs d'observer un nouveau type de résonance magnétique dans l'état excité [2], [3] et fondamental [4] d'un atome.

Rappelons brièvement le principe commun de ces expériences : soit $\mathrm{Oz}$ la direction du champ $H_{0}+H_{1} \cos \omega t$. L'excitation optique a une polarisation telle qu'elle fait apparaître instantanément un moment angulaire transversal $\mathbf{M}_{1}$ (perpendiculaire à $\mathrm{Oz}$ ) dans l'état excité ou fondamental du système atomique.

Supposons d'abord $H_{1}=0$. Les dipôles précessent autour de $H_{0}$ à la fréquence de Larmor $\omega_{0} / 2 \pi$ : crées uniformément au cours du temps et dans une direction fixe par l'excitation optique, ils se répartissent par suite dans le plan perpendiculaire à Oz. Soit $\tau$ la durée de vie (radiative ou de relaxation) du niveau atomique considéré. Si $\omega_{0} \tau \gg 1$, cette répartition est isotrope et l'aimantation transversale globale à l'état stationnaire $<\mathbf{M}_{\perp}>$ est identiquement nulle. Le pompage optique transversal d'une vapeur dans un champ statique $H_{0}$ n'est donc possible qu'en champ très bas, $\omega_{0} \tau \lesssim 1$ (effet Hanle dans l'état excité [6] et fondamental [7]).

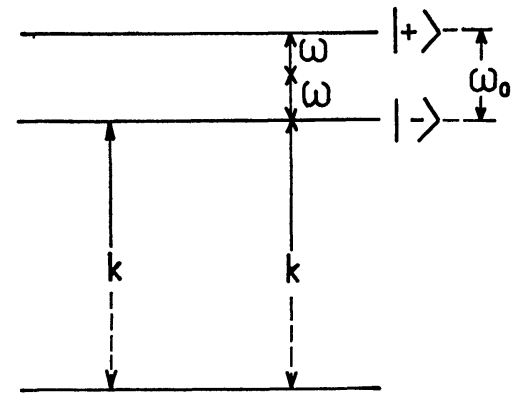

Fig. 4 .

La modulation du champ statique $H_{0}$ par le champ de radiofréquence $H_{1} \cos \omega t$ permet de réaliser le pompage optique transversal de la vapeur autrement qu'en champ très bas ; pour toute une série de valeurs de $H_{0}$ telles que $\omega_{0}=n \omega$ ( $n$ entier positif ou nul) il apparaît dans la vapeur une aimantation transversale globale $\left\langle\mathbf{M}_{\perp}\right\rangle$ effectuant autour de $H_{0}$ un mouvement périodique, de période $2 \pi / \omega$. Les caractéristiques de ces nouvelles résonances ont été étudiées en détail, le point essentiel étant que leur largeur ne dépend pas de $H_{1}$. 
L'apparition de ces nouvelles résonances se comprend aisément: la fréquence de Larmor étant maintenant modulée par $H_{1} \cos \omega t$, les dipôles ne se répartissent plus de façon isotrope dans le plan perpendiculaire à $H_{0}$, mais au contraire se groupent en paquets. La vitesse angulaire moyenne des dipôles est $\omega_{0}$; lors d'une période du champ $H_{1}$, $2 \pi / \omega$, ils ont tourné d'un angle $2 \pi \omega_{0} / \omega$; pour que l'effet de groupement en paquet soit cumulatif, et que $\left\langle M_{\perp}>\neq 0\right.$, il faut que cet angle soit un multiple de $2 \pi$; ceci permet de comprendre la condition de résonance : $\omega_{0}=n \omega$.

\section{B. INTERPRÉtation EN TERMES DE PHOtons DE} RADIOFRÉQUENCE. - Il ressort clairement de l'analyse précédente que l'apparition des nouvelles résonances est étroitement liée au fait que, pendant la durée de vie du niveau atomique étudié, le mouvement de précession du dipôle autour de $H_{0}$ n'est plus uniforme. Nous avons donné dans la première partie une interprétation de ce fait en termes quantiques : l'atome absorbe et réémet virtuellement des photons de radiofréquence. Le fait que ces transitions soient virtuelles et non relles entraîne que les niveaux d'énergie de l'atome ne sont pas élargis par interaction avec le champ $H_{1}$; on conçoit alors aisément que les résonances observées ne présentent aucun élargissement de radiofréquence. (Lorsque par contre la polarisation de $H_{1}$ est telle que des transitions réelles entre les sousniveaux Zeemann de l'atome sont possibles, comme c'est le cas par exemple dans l'expérience bien connue de double résonance [8], les niveaux atomiques et par suite les raies de résonance présentent un élargissement supplémentaire dû à $H_{1}$.)

Le problème a été abordé par Khodovoy [9] d'un point de vue différent : cet auteur interprète qualitativement et quantitativement les nouvelles résonances en faisant intervenir l'absorption simultanée d'un photon optique et de $n$ photons de radiofréquence; nous résumons ici brièvement son interprétation qualitative :

La présence d'une aimantation transversale globale dans la vapeur résulte de l'existence de cohérence hertzienne [10] entre les deux sous-niveaux Zeeman $\mid+>$ et $\mid->$. L'auteur suppose que la raie excitatrice de largeur $\Delta$, se décompose en une infinité d'ondes monochromatiques sans relation de phase les unes avec les autres, ayant une polarisation telle qu'elles peuvent exciter soit le niveau $\mid+>$ soit le niveau $\mid->$. Si $\omega_{0} \gg 1 / \tau$, il est impossible d'exciter à la fois les deux niveaux $\mid+>$ et $\mid->$ par la même onde monochromatique pour des raisons de conservation d'énergie. Il est cependant possible d'arriver à ce résultat avec l'aide du champ de radiofréquence : Considérons par exemple un photon optique dont l'énergie $k$ est telle qu'elle lui permet d'exciter le niveau $1->$ (fig. 4). Si $\omega_{0}=n \omega$, par absorption simul- tanée de ce mème photon optique et de $n$ photons de radiofréquence, le niveau $\mid+>$ pourra être également excité. La phase de l'onde optique de fréquence $k / 2 \pi$ intervient donc de la même manière dans l'excitation des états $\mid+>$ et $\mid->$. La différence de phase entre ces deux états ne dépend plus alors de la phase de l'onde optique : la cohérence hertzienne ainsi introduite subsiste lorsqu'on moyenne sur toutes les ondes optiques. Le phénomène est donc simple à comprendre pour une raie monochromatique. Pour tenir compte des conditions expérimentales réelles, il faut sommer l'effet précédent sur un spectre de fréquences optiques dont la largeur $\Delta$ est grande devant $\omega_{0}, \omega_{1}$ et $\omega$. On retrouve bien alors le résultat correct mais il devient difficile de décrire le phénomène global en faisant intervenir des absorptions simultanées d'un photon optique et de plusieurs photons de radiofréquence; pour $\Delta \gg \omega_{0}$, on montre en effet que les processus d'absorption et d'émission de photons de radiofréquence se compensent de sorte que globalement il ne subsiste aucune absorption (ou émission) réelle de ces photons.

Il ressort de l'analyse précédente que lors d'un€ excitation monochromatique, l'atome peut absorber simultanément un photon optique et plusieurs photons de radiofréquence. On peut alors se demander si la présence du champ $H_{1}$ modifie la probabilité d'absorption d'un photon optique lorsque la raie excitatrice a maintenant une largeur $\Delta$; et si par suite l'image habituelle du cycle de pompage optique (trois processus indépendants les uns des autres : absorption d'un photon optique, évolution propre, émission spontanée) reste toujours valable èn présence de $H_{1}$. Nous avons repris la théorie quantique du cycle de pompage optique en quantifiant non seulement l'onde lumineuse, mais aussi l'onde de radiofréquence [11]. On montre aisément que si la largeur de la raie est suffisamment grande, $\Delta \gg \omega, \omega_{0}$ et $\omega_{1}$, la probabilité $1 / T_{\mathrm{p}}$ d'excitation optique de l'atome est indépendante de $H_{1}$ (la durée du processus d'absorption $[10], 1 / \Delta$, est trop courte pour qu'un effet quelconque de $H_{1}$ puisse se manifester pendant ce même temps). L'image du cycle de pompage reste donc toujours valable et on peut se représenter le processus physique de la façon suivante: l'atome absorbe un photon optique, monte dans l'état excité, y reste une durée $\tau$ avant de retomber dans l'état fondamental par émission spontanée. Pendant le temps de vie $\tau$ de l'état excité, il absorbe et réémet virtuellement des photons de radiofréquence, ce qui rend sa précession de Larmor non uniforme et fait par suite apparaitre les nouvelles résonances.

Conclusion. - Nous avons présenté un calcul quantique du mouvement d'un dipôle magnétique dont on module la fréquence de Larmor. Tous les 
résultats du calcul classique sont retrouvés quantitativement. Nous avons pu montrer que l'apparition de fréquences latérales dans le mouvement $d u$ dipôle était due à des absorptions et réémissions virtuelles de photons de radiofréquence par le système atomique. Le fait que ces transitions soient virtuelles et non réelles permet de comprendre pourquoi certaines résonances magnétiques observées récemment dans des expériences de pompage optique transversal dans un champ magnétique modulé en amplitude ne présentent pas d'élargissement de radiofréquence.

\section{APPENDICE}

Calcul de l'élément de matrice $\left\langle\bar{n}_{+}\right|(\overline{n-q})_{-}>$. - Utilisant la relation (7), cet élément de matrice s'écrit :

$$
\begin{aligned}
& \left\langle\bar{n}_{+}\right|(\overline{n-q})>=\left\langle n\left|\mathrm{e}^{\lambda / \omega(a+-a}\right| n-q\right\rangle . \\
& \left\langle\bar{n}_{+} \mid(\overline{n-q})_{-}\right\rangle=\sum_{s}\left\langle n\left|(-1) s\left(\frac{\lambda}{\omega}\right)^{q+2 s} \frac{(a+)^{q+s} a^{s}}{(q+s) ! s !}\right| n-q>\right. \\
& =\sum_{s}(-1)^{s} \frac{\sqrt{n(n-1) \ldots(n-q-s)} v \overline{(n-q)(n-q-1) \ldots(n-q-s)}}{(q+s) ! s !}\left(\frac{\lambda}{\omega}\right)^{a+2_{s}} \text {. }
\end{aligned}
$$

Rappelons la propriété suivante [12] : Si deux opérateurs $A, B$ commutent avec $[A, B]$, on a :

$$
\mathrm{e}^{\mathrm{A}} \mathrm{e}^{\mathrm{B}}=\mathrm{e}^{\left\{A+B+\frac{1}{2}[A, B]\right\} .}
$$

Comme $\left[a, a^{+}\right]=1$, l'opérateur $\mathrm{e}^{\lambda_{\omega}^{\left(-a^{+}-a\right)}}$ peut donc s'écrire :

$$
\mathrm{e}^{\frac{\lambda}{\omega}\left(a^{+}-a\right)}=\mathrm{e}^{\frac{\lambda}{\omega^{+}} a^{+}} \mathrm{e}^{-\frac{\lambda}{\omega} a} \mathrm{e}^{-\frac{\lambda^{2}}{2 \omega^{2}}}
$$

$\lambda^{2} / \omega^{2}$ étant très petit devant l'unité, on prendra $\mathrm{e}^{-\lambda^{2} / \omega^{2}}=1$.

Développant en série les exponentielles, il vient : $<\bar{n}_{+} \mid \overline{n-q}->$

$=<n \mid\left[\sum_{m}\left(\frac{\lambda a^{+}}{\omega}\right)^{m} \frac{1}{m !}\right]\left[\sum_{s}\left(-\frac{\lambda a}{\omega}\right)^{s} \frac{1}{s !} \mid n-q>\right.$.

Les seuls termes non nuls de cette double somme sont tels que : $\left\langle n\left|\left(a^{+}\right)^{m} a^{s}\right| n-q>\neq 0\right.$ ce qui impose la relation : $n-m=n-q-s$, soit : $m=q+s$.

On a donc encore : ce qui n'est autre que le développement en série $[1, \S 7.5 .1]$ de $J_{q}(2 \lambda \sqrt{ } \bar{n} / \omega)$. On a donc ainsi

$$
<\bar{n}_{+} \mid(\overline{n-q})_{-}>=J_{q}(2 \lambda \sqrt{n} / \omega) .
$$

Comme $\lambda \sqrt{n} / \omega \simeq \lambda \sqrt{N} / \omega=\omega_{1} / 2 \omega$, on retrouve la relation (20) annoncée dans le paragraphe I-E-c

$$
\left.\left.\left\langle\bar{n}_{+}\right| \overline{n-q}\right)_{-}\right\rangle=J_{Q}\left(\omega_{1} / \omega\right) .
$$

Remarque. - Dans la représentation $x$, les états et $\overline{n_{+}}(\overline{n-q})_{-}$correspondent aux fonctions propres de l'oscillateur harmonique déplacées respectivement des quantités $+\lambda / \bar{V} \overline{2} \omega$ et $-\lambda / \sqrt{2} \omega$, soit

$u_{\mathrm{r}}\left(Q+\frac{\lambda}{\sqrt{2} \omega}\right)$ et $u_{n-q}\left(Q-\frac{\lambda}{\sqrt{2} \omega}\right)$ avec $Q=(m \omega)^{1 / 2} x$. La relation (25) s'écrit donc :

$\int_{-\infty}^{+\infty} U_{n}\left(Q+\frac{\lambda}{\sqrt{2} \omega}\right) U_{n-q}\left(Q-\frac{\lambda}{.2 \omega}\right) \mathrm{d} Q$

$$
=J_{q}\left(\frac{2 \lambda, n}{\omega}\right)
$$

Nous obtenons ainsi une relation entre les poly nômes d'Hermite $H_{n}$ et $H_{n-q}$ et la fonction de Bessel $J_{q}$ valable pour $n \gg 1$ :

$$
\begin{array}{r}
\int_{-\infty}^{+\infty} \frac{\mathrm{e}^{-\left(Q^{2}+\lambda_{2 \omega^{2}}^{\lambda^{2}}\right)} H_{n}\left(Q+\frac{\lambda}{\sqrt{2} \omega}\right) H_{n-q}\left(Q-\frac{\lambda}{\sqrt{2} \omega}\right) \mathrm{d} Q}{\sqrt{\pi 2^{2 n-\alpha} n !(n-q !)}} \\
=J_{q}\left(\frac{2 \lambda \vee \bar{n}}{\omega}\right) .
\end{array}
$$

\section{BIBLIOGRAPHIE}

[1] Angot (A.), Compléments de mathématiques, Édition de la Revue d'Optique, Paris.

[2] Favre (G. J.) et Geneux (E.), Physics Letters, 1964, $8, n^{\circ} 3,190$.

[3] Alexandrov (E. B.), Constantinov (O. B.), Perelli (B. I.) et Kнороvоч (B. A.), J. Exptl. Theoret. Phys., U. S. S. R., 1963, 45, 503.

[4] Polonsky (N.) et Cohen-Tannoudi (C.), $C . R$. Acad. Sc., Paris, 1965, 260, 5٪31.

[5] Glauber (Roy J.), Phys. Rev., 1963, 131, no 6, 27662788.

[6] Mitchell (A. C. G.) et Zemansky (M. W.), Resonance radiation and excited atoms, Cambridge University Press, London, 1934.
[7] Lehmann (J. C.) et Cohen-Tannoudi (C.), C. $R$. Acad. Sc., 1964, 258, 4463-4466.

[8] Brosser (J.), Thèse, Paris, 1951 ; Annales de Physique, $1952,7,622$.

[9] Khod ovoy (V. A.), J. Exptl. Theoret. Phys., U. S. S. R. January 1964, 46, 331-338.

[10] Cohen-Tannoudji (C.), Thèse, Paris, 1962 ; Annales de Physique, 1962, 7, 423 et 469 .

[11] Polonsky (N.), Thèse $3^{e}$ cycle, Paris, 1965.

[12] Messiah (A.), Mécanique quantique, Dunod, Paris, 1959, tome I, p. 375. 